Original Paper http://ajol.info/index.php/ijbcs http://indexmedicus.afro.who.int

\title{
Prévalence du pré-diabète et du diabète avec profil lipidique évalué, en milieu hospitalier urbain: étude rétrospective sur 3538 patients reçus au $\mathrm{CHU}$ Campus de Lomé
}

\author{
Kafui Codjo KOUASSI ${ }^{1,2^{*}}$, Lochina FÉTÉKÉ ${ }^{1,3}$ Santrao ETASSOLI ${ }^{4}$, \\ Efui Holaly GBEKLEY ${ }^{1}$ et D. Simplice KAROU ${ }^{1}$
}

${ }^{1}$ Faculté des Sciences (FDS) : Département de Biochimie, /Ecole Supérieure des Techniques Biologiques et Alimentaires (ESTBA)- Université de Lomé, Lomé-Togo.

${ }^{2}$ Centre Hospitalier Universitaire aaza Campus-Département de Biochimie, Lomé -Togo

${ }^{3}$ Faculté des sciences de la santé-Département des sciences fondamentales Université de Lomé, Lomé-Togo

${ }^{4}$ Institut National d'Assurance Médicale - Département des analyses bio-statistiques, Lomé-Togo

*Auteur correspondant ; E-mail : kafui.kouassi@gmail.com,ckouassi@univ-lome.tg ;

Téléphone : +22890077357/+22897386580

\section{RESUME}

Les sujets en état de pré-diabète sont exposés à un risque élevé de diabète et de dyslipidémies. La présente étude avait eu pour but de mesurer la prévalence du pré-diabète et du diabète chez des sujets reçus à l'hôpital, puis de déterminer le profil lipidique des pré-diabétiques. Il s'est agi d'une étude rétrospective à visée descriptive, portant sur 3538 patients âgés de 3 à 120 ans, reçus en 2013 au Centre Hospitalier Universitaire nommé Campus. La glycémie, la cholestérolémie totale, la HDL-Cholestérolémie, et la triglycéridémie ont été dosées sur l'automate SELECTRA PRO M. La non-HDL- Cholestérolémie a été calculée. La prévalence du pré-diabète a été déterminée suivant les critères de l'Organisation Mondiale de la Santé (OMS) et du «American Diabetes Association » (ADA). La prévalence du pré-diabète suivant les critères de l'OMS était de 8,1\% contre 21,5\% selon les critères de l'ADA. La prévalence du diabète était de 12,9\%. Elle était de 15,3\% chez les sujets âgés de 45 à 59 ans. Au sein de la population pré-diabétique 42,3 à 45,6\% avaient des dyslipidémies. La prévalence du pré-diabète à l'hôpital était importante. Une prise en charge de ce dernier permettrait de prévenir le diabète de type 2 et réduire les risques de dyslipidémies.

(c) 2020 International Formulae Group. All rights reserved.

Mots clés : Glycémie, dyslipidémies, pré-diabétiques, Togo.

\section{Prevalence of pre-diabetes and diabetes with evaluated lipid profile, in urban hospital: retrospective study on 3538 patients seen at the Lomé campus university hospital.}

\begin{abstract}
People with pre-diabetes are at high risk for diabetes and dyslipidemia. The aim of the present study was to measure the prevalence of pre-diabetes and diabetes in hospital patients, and then to determine the lipid profile of pre-diabetics. It was a retrospective descriptive study, involving 3538 patients aged 3 to 120 years, received in 2013 at the University Hospital Center called Campus. Blood glucose, total cholesterol, HDL-Cholesterolemia,
\end{abstract}


and triglyceridemia were measured on the SELECTRA PRO M automatic analyzer. Non-HDL-Cholesterolemia was calculated. The prevalence of pre-diabetes was determined according to the criteria of the World Health Organization (WHO) and the "American Diabetes Association" (ADA). The prevalence of pre-diabetes according to WHO criteria was $8.1 \%$ compared to $21.5 \%$ according to ADA criteria. The prevalence of diabetes was $12.9 \%$. It was $15.3 \%$ in subjects aged 45 to 59 years. In the pre-diabetic population 42.3 to $45.6 \%$ had dyslipidemia. The prevalence of pre-diabetes in hospital was high. Management of the latter would prevent type 2 diabetes and reduce the risk of dyslipidemia.

(c) 2020 International Formulae Group. All rights reserved.

Keywords: Glycemia, dyslipidemia, pre-diabetics, Togo.

\section{INTRODUCTION}

En 2017, en Afrique, 15,9 millions de personnes vivaient avec le diabète et 42,9 millions âgés de 18 à 99 ans étaient en état de pré-diabète. Selon la Fédération Internationale du Diabète (FID) l'Afrique est la région avec le pourcentage le plus élevé de personnes non diagnostiquées, soit $70 \%$ des diabétiques. Avec une proportion plus importante de diabète du type 2. Au Benin ils étaient à $98,8 \%$ contre $0,9 \%$ du diabète de type 1 (Fagbemi et al., 2017 ; Suvi et al., 2017). Le pré-diabète se caractérise par des anomalies de la glycorégulation avec une glycémie à jeun supérieure ou égale à $6,1 \mathrm{mmol} / \mathrm{l}(110 \mathrm{mg} / \mathrm{dl})$, mais inférieure à $7,0 \mathrm{mmol} / \mathrm{l}(126 \mathrm{mg} / \mathrm{dl})$ selon l'Organisation Mondiale de la Santé (OMS). Selon l'American Association of Diabetes (ADA), il est défini par une glycémie à jeun supérieure ou égale à $5,6 \mathrm{mmol} / \mathrm{l}(100 \mathrm{mg} / \mathrm{dl})$ mais inférieure à $7,0 \mathrm{mmol} / \mathrm{l}$. Les sujets en état de pré-diabète sont exposés à un risque élevé de diabète qui est l'une des 4 maladies nontransmissibles majeures, ayant causé environ 4 millions de décès en 2017 (ADA, 2015 ; Shen et al., 2016). En 2010, selon l'enquête de surveillance des facteurs de risque des maladies chroniques (STEPS)-Togo de l'OMS, la prévalence de l'hyperglycémie était de 2,6\% dans les populations de 15 à 64 ans (Agoudavi et al., 2012). En 2014, selon l'OMS, la prévalence du diabète chez les sujets de plus de 18 ans était de 5\% (WHO, 2018). Ces données ne permettent pas de connaître spécifiquement, la prévalence du pré-diabète, dans un contexte où, l'approche de gestion de risques et de prévention en santé publique amène les décideurs à s'intéresser aux situations intermédiaires entre la normo-glycémie et le diabète (Schlienger, 2014). En 2013 la prévalence du pré-diabète aurait évoluée, et il n'existait pas de données sur la prévalence du pré-diabète et du diabète en milieu hospitalier urbain au Togo. En plus le pré-diabète et les dyslipidémies font parties des critères caractéristiques du syndrome métabolique. Les dyslipidémies constituent des causes de complication du diabète. (Koevi, 2014 ; Suvi et al., 2017). La présente étude avait eu pour but de mesurer la prévalence du pré-diabète et du diabète chez des sujets reçus à l'hôpital, puis de déterminer le profil lipidique des prédiabétiques.

\section{MATERIEL ET METHODES}

Il s'est agi d'une étude rétrospective à visée descriptive, portant sur 3640 sujets reçus au sein du service des laboratoires du CHU Campus entre le 12 mars et le 10 décembre 2013. Ont été inclus dans l'étude 3538 patients de tout âge, disposant dans les registres d'une prescription de glycémie à jeun, de la cholestérolémie totale (CT), de l'HDL (High Density Lipoprotein)-Cholestérolémie (HDLC) et de la triglycéridémie (TG). Les patients étaient externes ou hospitalisés au CHU Campus, avec leurs données démographiques relevées. Ils étaient à jeun d'au moins 11 à 12 heures et avaient fait l'objet d'un prélèvement sanguin après recueil verbal d'un consentement éclairé. L'étude était réalisée en accord avec la déclaration de bioéthique d'Helsinki et conformément aux exigences de la norme ISO 15189 version 2012. L'anonymat des données et le respect de la confidentialité ont donc été appliqués. 


\section{Examens Biochimiques}

Les examens de glycémie, de CT, de TG et d'HDL-C étaient réalisés sur l'automate SELECTRA PRO M de Vital Scientifics (Pays Bas) avec les réactifs Elitech (France) programmés suivant les instructions du fabricant. Les valeurs de référence utilisées pour le bilan lipidique étaient celles du NCEP (National Cholesterol Education Program) du National Institutes of Health (NIH) des EtatsUnis (NIH, 2001). Les méthodes biochimiques utilisées étaient du mode Trinder $\left(\mathrm{H}_{2} \mathrm{O}_{2}-\right.$ Péroxydase : EC 1.11.1): la glycémie avec la méthode de la glucose oxydase (EC 1.1.3.4) ; la cholestérolémie avec la cholestérol oxydase (EC 1.1.3.6) et la triglycéridémie par la glycérol-3 phosphate oxydase (EC 1.1.3.21). L'HDL-C a été obtenu par dosage dans le surnageant après précipitation des chylomicrons et LDL (Low Density Lipoprotein)/VLDL (Very Low Density Lipoprotein)-cholestérol par addition du phosphotungstate de magnésium. Ce surnageant a été obtenu par séparation après centrifugation à 4000 tours/mn (3095 g) pendant $10 \mathrm{mn}$ sur une centrifugeuse de marque Jouan de Thermo-Scientifics. Ces mesures spectrophotométriques étaient standardisées grâce à des étalons (étalonnage à deux points) définis par rapport à la méthode de référence «Isotopic Dilution - Mass Spectrometry (ID-MS) ».

\section{Critères de diagnostics}

La prévalence du pré-diabète a été déterminée suivant les critères de l'OMS (glycémie $\geq 6,1 \mathrm{mmol} / 1$ et $<7,0 \mathrm{mmol} / \mathrm{l}$ ) et de l'ADA (glycémie $\geq 5,6 \mathrm{mmol} / \mathrm{l}$ et $<7,0$ mmol/l) (Suvi et al., 2017 ; ADA, 2018). Le critère de glycémie $\geq 7,0 \mathrm{mmol} / \mathrm{l}$ a été utilisé pour évaluer la prévalence du diabète. Les critères de dyslipidémie utilisés étaient : TG $\geq$ $1,7 \mathrm{mmol} / \mathrm{l}(150 \mathrm{mg} / \mathrm{dl}), \mathrm{CT} \geq 5,2 \mathrm{mmol} / \mathrm{l}(200$ $\mathrm{mg} / \mathrm{dl}$ ) et le non-HDL-Cholestérol (CT HDL-C) > 4,1 mmol/l $(160 \mathrm{mg} / \mathrm{dl})$. Pour évaluer un risque élevé de pathologies cardiovasculaires, le critère de $\mathrm{TG} \geq 2,3 \mathrm{mmol} / \mathrm{l}$ (200 mg/dl) a été utilisé (ADA, 2015 ; Shen et al., 2016). Le facteur de risque âge $\geq 45$ ans pour les hommes et $\geq 55$ ans pour les femmes a été pris en compte (NIH, 2001 ; Jellinger et al., 2017).

\section{Analyses statistiques}

Les paramètres sociodémographiques et biologiques des patients ont été relevés et les données analysées par le logiciel R (Microsoft version 3.4 de 2018). Les résultats ont été exprimés en moyenne \pm écart-type pour les données à distribution normale et en médiane avec les quartiles pour les données nonparamétriques. Le test de KolmogorovSmirnov a été utilisé pour s'assurer du type de distribution des données. Les prévalences ont été exprimées en $\%$ avec les intervalles de confiances à 95\%. Les principaux tests statistiques utilisés étaient : le test du Chi-2 et le test exact de Fischer. Le seuil de significativité utilisé était de 5\%.

\section{RESULTATS}

\section{Aspects démographiques et caractéristiques diverses}

Les sujets de l'étude étaient majoritairement des sujets externes $(72,7 \%)$. Les autres étaient des patients hospitalisés dans les services de neurologie $(8,4 \%)$, d'HépatoGastro-Entérologie (7,4\%), de Médecine interne $(6,8 \%)$ et de cardiologie $(4,7 \%)$. Ils étaient 2021 (57,2\%) femmes versus 1517 $(42,8 \%)$ hommes. Les âges extrêmes étaient de 3 à 120 ans. Les sujets étaient majoritairement âgés de 19 à 44 ans (1309 soit 37,0\%) et de 45 à 59 ans (1172 soit 33,1\%).

La glycémie était dosée chez les 3538 sujets. Les résultats des examens du bilan lipidique étaient disponibles chez 1815 sujets $(51,3 \%)$ pour la CT ; $1699(48,0 \%)$ pour l'HDL-C et $1792(50,6 \%)$ à propos de la TG.

\section{Prévalence du pré-diabète et du diabète}

L'OMS et l'ADA ont établi des critères d'identification des sujets en situation de prédiabète. La prévalence du pré-diabète était de 
$8,1 \%$ selon les critères de l'OMS avec une glycémie moyenne de $6,4 \pm 0,2 \mathrm{mmol} / \mathrm{l}$ $(116,0 \pm 4,0 \mathrm{mg} / \mathrm{dl})$. Celle selon les critères de l'ADA était de $21,5 \%$ avec une glycémie moyenne de $5,9 \pm 0,3 \mathrm{mmol} / \mathrm{l} \quad(108,0 \pm 6,0$ $\mathrm{mg} / \mathrm{dl})$. La prévalence du diabète était de 12,9\%. Le Tableau 1 présente les détails des prévalences du pré-diabète et du diabète par tranche d'âge.

Il ressort de ce tableau que la prévalence du pré-diabète selon les critères de l'OMS et de l'ADA était plus élevée au sein de la tranche d'âge [45,60 [ soit respectivement $10,2 \%$ et $25,5 \%$; que dans la tranche d'âge [19,45[ soit $6,2 \%$ et $18,0 \%$. La prévalence du diabète était de 15,3\% au sein de la tranche d'âge [45-60[, mais de 7,5\% chez les sujets âgés de 19 à 44 ans. Cette prévalence du diabète était encore plus élevée chez les personnes âgées $[60,120]$ soit $19,2 \%$.

\section{Profil lipidique des pré-diabétiques selon des critères de l'OMS et de l'ADA}

Dans la population générale de ceux qui ont réalisé les examens du bilan lipidique
$33,8 \%(\mathrm{n}=613)$ avaient une CT $\geq 5,2 \mathrm{mmol} / \mathrm{l}$, $35,7 \%(\mathrm{n}=608)$ une Non-HDL $\mathrm{C}>4,1 \mathrm{mmol} / \mathrm{l}$ et $13,8 \%(\mathrm{n}=247)$ une $\mathrm{TG} \geq 1,7 \mathrm{mmol} / \mathrm{l}$. Le critère Non-HDL $\mathrm{C}>4,1 \mathrm{mmol} / \mathrm{l}$ a révélé que chez les femmes et hommes âgés respectivement de 55 et 45 ans au moins, les sujets affectés par cette dyslipidémie étaient significativement plus important :

- Femmes : 51,4\% ( $\mathrm{n}=196)$ versus $48,6 \%$ $(\mathrm{n}=186), \mathrm{P}<0,001$;

- Hommes : (72,2\% (163) versus $27,8 \%$ (63) ; $\mathrm{P}<0,002$.

Les sujets au nombre de 288 et 760 respectivement sujets pré-diabétiques suivant les critères de l'OMS et d'ADA ont pu réaliser les examens du bilan lipidique dans des proportions différentes. La cholestérolémie était l'examen le plus réalisé, soit 171(59,3\%) sujets des pré-diabétiques OMS et $451(62,6 \%)$ des pré-diabétiques ADA. Les données détaillées en fonction des tranches d'âges sont présentées dans le Tableau 2.

Il ressort de ce tableau que les sujets au sein des tranches d'âge [19,45[ et [45,60[ étaient plus affectés par les dyslipidémies.

Tableau 1 : Prévalence par tranche d'âge du pré-diabète selon l'OMS et ADA et du diabète

\begin{tabular}{|c|c|c|c|c|c|c|c|c|c|c|}
\hline \multirow{2}{*}{$\begin{array}{l}\text { Anomalies du } \\
\text { métabolisme } \\
\text { glucidique }\end{array}$} & \multicolumn{2}{|c|}{$\begin{array}{l}3,120] \\
n=3538\end{array}$} & \multicolumn{2}{|c|}{$\begin{array}{l}{[3,19]} \\
n=152\end{array}$} & \multicolumn{2}{|c|}{$\begin{array}{l}{[19,45[} \\
n=1309\end{array}$} & \multicolumn{2}{|c|}{$\begin{array}{l}{[45,60[} \\
n=1172\end{array}$} & \multicolumn{2}{|c|}{$\begin{array}{c}{[60,120]} \\
n=905\end{array}$} \\
\hline & $\begin{array}{c}\text { OMS } \\
\%(\mathrm{n}) \\
\text { IC }\end{array}$ & $\begin{array}{c}\text { ADA } \\
\%(\mathrm{n}) \\
\text { IC }\end{array}$ & $\begin{array}{c}\text { OMS } \\
\%(\mathrm{n}) \\
\text { IC }\end{array}$ & $\begin{array}{c}\text { ADA } \\
\%(\mathrm{n}) \\
\text { IC }\end{array}$ & $\begin{array}{c}\text { OMS } \\
\%(\mathrm{n}) \\
\text { IC }\end{array}$ & $\begin{array}{c}\mathrm{ADA} \\
\%(\mathrm{n}) \\
\mathrm{IC}\end{array}$ & $\begin{array}{c}\text { OMS } \\
\%(\mathrm{n}) \\
\text { IC }\end{array}$ & $\begin{array}{c}\mathrm{ADA} \\
\%(\mathrm{n}) \\
\mathrm{IC}\end{array}$ & $\begin{array}{c}\text { OMS } \\
\%(\mathrm{n}) \\
\text { IC }\end{array}$ & $\begin{array}{c}\mathrm{ADA} \\
\%(\mathrm{n}) \\
\mathrm{IC}\end{array}$ \\
\hline Pré-diabète & $\begin{array}{c}8,1 \\
(288) \\
7,3- \\
9,1\end{array}$ & $\begin{array}{l}21,5(760) \\
20,1-22,8\end{array}$ & $\begin{array}{l}3,9 \\
(6) \\
1,8- \\
8,3\end{array}$ & $\begin{array}{l}12,5 \\
(19) \\
8,2- \\
18,7\end{array}$ & $\begin{array}{c}6,2 \\
(81) \\
5,0- \\
7,6\end{array}$ & $\begin{array}{l}18,0 \\
(236) \\
16,0- \\
20,2\end{array}$ & $\begin{array}{c}10,2 \\
(119) \\
8,5- \\
12,0\end{array}$ & $\begin{array}{c}25,5 \%(299) \\
23,1-28,1\end{array}$ & $\begin{array}{c}9,1 \\
(82) \\
7,5- \\
11,1\end{array}$ & $\begin{array}{l}22,7 \\
(206) \\
20,1- \\
25,6\end{array}$ \\
\hline Diabète & & $\begin{array}{l}(459) \\
-19,0\end{array}$ & & & $\begin{array}{r}7,5 \% \\
3,9\end{array}$ & & & $\begin{array}{l}3(119) \\
, 2-17,5\end{array}$ & $\begin{array}{r}19,2 \% \\
14,5\end{array}$ & $\begin{array}{l}(174) \\
22,3\end{array}$ \\
\hline
\end{tabular}

IC : Intervalle de Confiance à 95\% / 95\% Confidence Interval 
Tableau 2 : Dyslipidémies par tranche d'âge chez les sujets pré-diabétiques selon l'OMS et ADA.

\begin{tabular}{|c|c|c|c|c|c|c|c|c|c|c|}
\hline \multirow[b]{2}{*}{$\begin{array}{c}\text { Critères de } \\
\text { dyslipidémie }\end{array}$} & \multicolumn{2}{|c|}{$[3,120]$} & \multicolumn{2}{|c|}{$[3,19]$} & \multicolumn{2}{|c|}{$[19,45[$} & \multicolumn{2}{|c|}{$[45,60[$} & \multicolumn{2}{|c|}{$[60,120]$} \\
\hline & $\begin{array}{c}\text { OMS } \\
\% \\
(\mathrm{n})\end{array}$ & $\begin{array}{c}\text { ADA } \\
\% \\
(\mathrm{n})\end{array}$ & $\begin{array}{l}\text { OMS } \\
\% \\
(\mathrm{n})\end{array}$ & $\begin{array}{c}\text { ADA } \\
\% \\
(\mathrm{n})\end{array}$ & $\begin{array}{l}\text { OMS } \\
\% \\
(\mathrm{n})\end{array}$ & $\begin{array}{c}\text { ADA } \\
\% \\
(\mathrm{n})\end{array}$ & $\begin{array}{c}\text { OMS } \\
\% \\
(\mathrm{n})\end{array}$ & $\begin{array}{c}\text { ADA } \\
\% \\
(\mathrm{n})\end{array}$ & $\begin{array}{c}\text { OMS } \\
\% \\
\text { (n) }\end{array}$ & $\begin{array}{c}\mathrm{ADA} \\
\% \\
(\mathrm{n})\end{array}$ \\
\hline $\begin{array}{l}\mathbf{C T} \geq \mathbf{5 , 2} \\
\mathbf{m m o l} / \mathbf{l} \\
\text { OMS }: \mathrm{n}=171 \\
\text { ADA }: \mathrm{n}= \\
451\end{array}$ & $\begin{array}{c}45,6 \% \\
(78)\end{array}$ & $\begin{array}{c}42,3 \% \\
(191)\end{array}$ & $\begin{array}{c}0 \\
(0)\end{array}$ & $\begin{array}{l}2,1 \\
(4)\end{array}$ & $\begin{array}{l}20,5 \\
(16)\end{array}$ & $\begin{array}{l}21,9 \\
(42)\end{array}$ & $\begin{array}{l}51,4 \\
(40)\end{array}$ & $\begin{array}{l}45,1 \\
(86)\end{array}$ & $\begin{array}{l}28,2 \\
(22)\end{array}$ & $\begin{array}{l}30,9 \\
(59)\end{array}$ \\
\hline $\begin{array}{l}\text { HDL-C }<\mathbf{1 , 0} \\
\mathbf{m m o l} / \mathbf{l} \\
\text { OMS : } \mathrm{n}=163 \\
\text { ADA: } \mathrm{n}=420\end{array}$ & $\begin{array}{c}41,7 \% \\
(68)\end{array}$ & $\begin{array}{c}44,1 \% \\
(185)\end{array}$ & $\begin{array}{l}2,9 \\
(2)\end{array}$ & $\begin{array}{l}2,7 \\
(5)\end{array}$ & $\begin{array}{l}33,8 \\
(23)\end{array}$ & $\begin{array}{l}25,4 \\
(47)\end{array}$ & $\begin{array}{l}36,8 \\
(25)\end{array}$ & $\begin{array}{l}43,2 \\
(80)\end{array}$ & $\begin{array}{l}26,5 \\
(18)\end{array}$ & $\begin{array}{l}28,7 \\
(53)\end{array}$ \\
\hline $\begin{array}{l}\text { Non-HDL-C } \\
>\mathbf{4 , 1} \mathbf{~ m m o l} / \mathbf{l} \\
\text { OMS }: \mathrm{n}=163 \\
\text { ADA }: \mathrm{n}=420\end{array}$ & $\begin{array}{c}43,5 \% \\
(71)\end{array}$ & $\begin{array}{l}43,2 \% \\
(181)\end{array}$ & $\begin{array}{c}0 \\
(0)\end{array}$ & $\begin{array}{l}2,2 \\
(4)\end{array}$ & $\begin{array}{l}23,9 \\
(17)\end{array}$ & $\begin{array}{l}23,3 \\
(42)\end{array}$ & $\begin{array}{l}47,8 \\
(34)\end{array}$ & $\begin{array}{l}44,7 \\
(81)\end{array}$ & $\begin{array}{l}28,3 \\
(20)\end{array}$ & $\begin{array}{l}29,8 \\
(54)\end{array}$ \\
\hline $\begin{array}{l}\text { TG } \geq \mathbf{1 , 7} \\
\mathbf{m m o l} / \mathbf{l} \\
\text { OMS }: n=168 \\
\text { ADA }: n=447\end{array}$ & $\begin{array}{c}12,5 \% \\
(21)\end{array}$ & $\begin{array}{c}15,6 \% \\
(70)\end{array}$ & $\begin{array}{c}0 \\
(0)\end{array}$ & $\begin{array}{l}1,4 \\
(1)\end{array}$ & $\begin{array}{l}23,8 \\
(5)\end{array}$ & $\begin{array}{l}28,6 \\
(20)\end{array}$ & $\begin{array}{l}52,3 \\
(11)\end{array}$ & $\begin{array}{l}44,3 \\
(31)\end{array}$ & $\begin{array}{c}23,8 \\
(5)\end{array}$ & $\begin{array}{l}25,7 \\
(18)\end{array}$ \\
\hline
\end{tabular}

\section{DISCUSSION}

Les prévalences du pré-diabète et du diabète n'ont pas été établies dans la population qui fréquente le CHU Campus, situé dans la région sanitaire « Lomé-commune » du Togo. Celle du diabète dans la population générale du Togo était néanmoins établie en 2010 en utilisant les critères de présence d'une hyperglycémie $(\geq 6,1 \mathrm{mmol} / \mathrm{l})$ ou d'un diabète diagnostiqué au glucomètre (Agoudavi et al., 2012). Les résultats obtenus dans la présente étude ont montré que la prévalence du prédiabète suivant les critères de l'OMS était de $8,1 \%$ et de $21,5 \%$ selon les critères de l'ADA. Il s'agit également d'une mise à jour des données de prévalence du diabète et de la première détermination de la prévalence du pré-diabète auprès d'une population urbaine reçue à l'hôpital, car 72,7\% des sujets étaient externes. L'utilisation de la fraction Non-HDLC a permis de s'affranchir de la formule de Friedewald pour le LDL-C qui comporte des limites pour les patients ayant une triglycéridémie supérieure ou égale à 4,6 $\mathrm{mmol} / \mathrm{l}(400 \mathrm{mg} / \mathrm{dl})$. En effet, la détermination du LDL-C par la formule de Friedewald devient non fiable pour les triglycéridémie entre $2,3 \mathrm{mmol} / \mathrm{l}(200 \mathrm{mg} / \mathrm{dl})$ et $4,6 \mathrm{mmol} / \mathrm{l}$ (400 mg/dl) selon l'association américaine d'endocrinologie clinique (Jellinger et al., 2017). Les seuils d'âges à risque de 45 et 55 ans étaient également utilisés par Assoumanou et al. (2012) dans l'évaluation du profil lipidique et du syndrome métabolique chez 270 adultes au Bénin.

Les résultats de l'étude étaient concordants avec ceux obtenus au Bengladesh, un pays à ressources limitées comme le Togo, dans une population urbaine âgée de plus de 35 ans. En 2011, la prévalence du diabète était de $15,2 \%$ dans ce pays (Akter et al., 2014). Elle était de 15,3\% chez les sujets âgés de 45 à 59 ans mais de 7,5\% chez les sujets âgés de 19 à 44 ans dans la présente étude. La prévalence du diabète était plus élevée dans la tranche d'âge 45 à 59 ans, car il a été établi que le diabète se 
révèle souvent après 40 ans et surtout dans un contexte de surcharge pondérale. Le prédiabète pouvait s'installer bien avant (Goldenberg et Punthakee, 2013). Cette même étude d'Akter et al. (2014) a établi que la prévalence du pré-diabète en milieu urbain était de $18,8 \%$ en utilisant le critère d'ADA contre $21,5 \%$ dans cette étude. Cette dernière prévalence était plus élevée car les patients étaient recrutés à partir d'une population déjà malade. Nwatu et al. (2016) au Nigéria en 2013, en milieu rural avaient obtenu une prévalence du pré-diabète de $9,3 \%$ chez des sujets âgés d'au moins 18 ans en utilisant les critères de l'OMS, alors qu'ici en milieu hospitalier et urbain, une prévalence de $8,1 \%$ avec le même critère d'OMS a été obtenue. Ces prévalences similaires pour des recrutements faits en milieu urbains à l'hôpital ou non ; ou en milieu rural, indiqueraient que le niveau de prévalence dépendrait aussi, du mode d'alimentation caractérisé par la consommation des aliments à index glycémique (IG) supérieur à 70 , et du manque d'activité physique régulière (Houlbert et al., 2008).

Concernant la survenue des dyslipidémies chez les pré-diabétiques de cette étude, il a été montré que $45,6 \%$ de ces sujets avaient une cholestérolémie $\geq 5,2 \mathrm{mmol} / \mathrm{l}$ et $12,5 \%$ une triglycéridémie $\geq 1,7 \mathrm{mmol} / 1$. En effet, il est établi que la moitié du cholestérol de l'organisme est produite par synthèse et le reste grâce à la ration alimentaire provenant surtout de la consommation du jaune d'œuf, de la viande et des abats comprenant le foie et le cerveau. Cette synthèse est faite à partir de l'acétyl-CoA, existant en quantité importante chez les pré-diabétiques qui développeraient une faible capacité de stockage du glycogène (Botham, 2011). Chez les pré-diabétiques âgés d'au moins 20 ans, Bishwajit et al. (2016) au Bengladesh en 2009 ont trouvé que 13,2\% avaient une $\mathrm{CT} \geq 5,2 \mathrm{mmol} / \mathrm{l}$ avec $42,1 \%$ qui avaient une $\mathrm{TG} \geq 1,7 \mathrm{mmol} / 1$. Chez les prédiabétiques de cette étude, c'est plutôt la proportion de ceux qui avaient une cholestérolémie $\geq 5,2 \mathrm{mmol} / \mathrm{l}$ qui étaient plus importante. En effet, une triglycéridémie élevée pourrait provenir également d'une consommation excessive de fructose : la lipogenèse des acides gras est plus forte lorsque le saccharose remplace le glucose, car le fructose contourne le point de contrôle de la phospho-fructokinase au cours de la glycolyse et afflue dans la voie lipogénique (Botham, 2011). Abessolo et al. (2012) ont trouvé chez 528 diabétiques en 2010 qu'une activité sportive hebdomadaire étaient significativement associé à la baisse du taux des TG. Une investigation des habitudes alimentaires et pratiques d'activités physiques permettraient de confirmer le type de métabolisme en accord avec les résultats obtenus.

Les résultats de l'étude montraient que 43,2 à $43,5 \%$ des pré-diabétiques, respectivement suivant les critères OMS et ADA avaient des concentrations des NonHDL-C > 4,1 mmol/l (tableau 2). En effet, les sociétés savantes «American Association of Clinical Endocrinologists (AACE) et «American College of Endocrinology » recommandent l'utilisation du Non-HDL-C à la place du LDL-C, car la Non-HDL-C serait le paramètre le plus prédictif des risques cardiovasculaires (Jellinger et al., 2017). Ce critère a également permis d'attester grâce à cette étude que le facteur "âge $\geq 45$ chez les hommes et $\geq 55$ ans chez les femmes» constitue un risque de survenue de plus de dyslipidémie. Cela était déjà évoqué par Liu et al. (2005) dans Diabetes care. Ghodsi et al. (2017) dans une population iranienne âgée de 24 à 65 ans a établi en 2007 un lien significatif entre l'augmentation de la Non-HDL-C et la survenue du syndrome métabolique dont le critère de base est le pré-diabète. L'association entre la Non-HDL-C et le syndrome métabolique sera évaluée dans une prochaine étude qui devrait s'étendre aux patients fréquentant les milieux hospitaliers ruraux des autres régions sanitaires du Togo.

\section{Conclusion}

Cette étude a permis d'attirer l'attention sur la nécessité de se préoccuper de la prévalence élevée du pré-diabète en milieu hospitalier pour prévenir le diabète grâce à des actions de sensibilisation à l'endroit des 
patients reçus à l'hôpital. Au sein des sujets pré-diabétiques, la proportion des dyslipidémies était élevée. $\mathrm{La} \mathrm{TG} \geq 2,3 \mathrm{mmol} / 1$ pourrait être utilisée pour mettre l'accent sur un éventuel risque cardiovasculaire élevé.

\section{CONFLIT D'INTERETS}

Les auteurs déclarent n'avoir aucun conflit d'intérêts concernant les données publiées dans cet article.

\section{CONTRIBUTIONS DES AUTEURS}

KCK : initiateur de l'étude, validation technique et biologique des résultats des examens réalisés, recueil des données et rédaction de l'article; LF : co-initiateur de l'étude, lecture du projet d'article, relecture de l'article ; SE : analyse statistique des données ; EHG: rédaction du projet d'article; DSK : relecture et validation finale de l'article à publier.

\section{REMERCIEMENTS}

$\mathrm{Au}$ regretté Prof. Yvon Akuété SEGBENA pour avoir accepté et facilité la réalisation de cette étude, afin d'améliorer la qualité des prestations de conseils fournis aux patients des services de laboratoire et des unités d'hospitalisation du CHU Campus. Egalement à Mme Eméfa APELETE et à $\mathrm{M}$. Atandji DONYO pour leur assistance dans la collecte des données.

\section{REFERENCES}

Abessolo FO, Bekale S, Jose F, Moutsinga Y, Milama EN.2012. Profil lipidique, cinétique d'apparition des diènes conjugués et activité paraoxonase- 1 chez le diabétique de type 2 à Libreville au Gabon. Int. J. Biol. Chem. Sci., 6(2): 572581.

DOI:

http://dx.doi.org/10.4314/ijbcs.v6i2.2

Agoudavi K, Adjoh KS, Afanvi K, Agbétsiafa K, Amédegnato D, Baba AB, Dogbé KS Tamékloe T. 2010. Rapport final de l'enquête STEPS Togo en 2010. Service des maladies non-transmissibles de la division d'épidémiologie. [https://www.who.int/ncds/surveillance/s teps/2010STEPS_Report_Togo_FR.pdf].
Ministère de la santé, 2012 (consulté le 20 mai 2020).

Akter S, Rahman M, Krull-Abe S, Sultana P. 2014. Prevalence of diabetes and prediabetes and their risk factors among Bangladeshi adults: a nationwide survey. Bull World Health Organ, 92(3): 204213A.

DOI: https://doi.org/10.2471/BLT.13.128371

American Diabetes Association. 2015. Classification and diagnosis of diabetes: standards of Medical Care in Diabetes. Diabetes Care, 38(1): S8-S16. DOI: https://doi.org/10.2337/dc15-S005

American Diabetes Association.2018. Glycemic Targets: Standards of Medical Care in Diabetes-2018. Diabetes Care, 41(1): $\quad$ S55-S64. DOI: https://doi.org/10.2337/dc18-S006

Assoumanou MG, Dovonou AC, Ngome MR, Akpona SA. 2012. Prévalence du syndrome métabolique chez les sujets hypertendus adultes dans les formations sanitaires de Parakou (Bénin). Int. J. Biol. Chem. Sci., 6(4): 1419-1427. DOI : http://dx.doi.org/10.4314/ijbcs.v6i4.

Bhowmik B, Siddiquee T, Mujumder A, Afsana F, Ahmed T, et al. 2018. Serum lipid profile and its association with diabetes and prediabetes in a rural Bangladeshi population. Int. J. Environ. Res. Public Health, 15(9): 1944. DOI: https://doi.org/10.3390/ijerph15091944

Botham K, Mayes P. 2011. Synthèse, transport et excrétion du cholestérol. Biosynthèse des acides gras. In Biochimie Illustrée de Harper, De boeck (ed). Nouveaux Horizon : Paris ; 193-204, 224-233.

Fagbemi KA, Azonbakin S, Adjagba M, BabaMoussa L, Laleye A.2017. Aspects épidémiologiques du diabète de type 1 à la banque d'insuline de Cotonou (Bénin). Int. J. Biol. Chem. Sci., 11(3): 1085-1095. DOI :

https://dx.doi.org/10.4314/ijbcs.v11i3.13

Ghodsi S, Meysamie A, Abbasi M, Ghalehtaki R, Esteghamati A, Malekzadeh MM, Asgari F, Gouya MM. 2017. Non-highdensity lipoprotein fractions are strongly associated with the presence of metabolic 
syndrome independent of obesity and diabetes: a population-based study among Iranian adults. J. Diabetes \& Metab. Disord, 16: $25 . \quad$ DOI: https://doi.org/10.1186/s40200-0170306-6

Houlbert A, Molénat V, Nérin E, Périault A, Soleille C, Souccar T, Tremblais P. 2008. La Meilleure façon de Manger. Le Premier Guide Alimentaire Basé sur la Science, Thierry Soucar (ed). Marabout Santé : Vergèze.

Jellinger $\mathrm{P}$, Handelsman $\mathrm{Y}$, Rosenblit $\mathrm{P}$, Bloomgarden Z, Fonseca V, Garber A, Grunberger G, Guerin C, Bell D, Mechanick J, Pessah-Pollack R, Wyne K, Smith D, Brinton E, Fazio S, Davidson M. 2017. American association of clinical endocrinologists and american college of endocrinology guidelines for management of dyslipidemia and prevention of cardiovascular disease. Endocrine practice, 23(2): 1-87. DOI: https://doi.org/10.4158/EP171764.APPG L

Koevi KKA, Millogo V, Ouedraogo M, Ouedraogo GA. 2014. Diagnostic des causes de complication du diabète et des méthodes de prévention à BoboDioulasso, au Burkina Faso. Int. J. Biol. Chem. Sci., 8(6): 2709-2720. DOI: http://dx.doi.org/10.4314/ijbcs.v8i6.30

Liu J, Sempos C, Donahue R, Dorn J, Trevisan M, Grundy SM. 2005. Joint distribution of non-HDL and LDL cholesterol and coronary heart disease risk prediction among individuals with and without diabetes. Diabetes Care, 28: 1916 -1921.
National Institutes of Health. 2001. Expert panel on detection, evaluation, and treatment of high blood cholesterol in adults: executive summary of the third report of the national cholesterol education program (NCEP). JAMA, 285(19): 2486-2497. DOI: https://doi.org/10.1001/jama.285.19.248 6

Nwatu C, Ofoegbu E, Unachukwu C, Young E, Okofor CI, Okoli CE. 2016. Prevalence of prediabetes and associated risk factors in a rural Nigerian community. Int $J$ Diabetes Dev Ctries, 36 (2): 197-203.

Schlienger J-L. 2014. Diabète. In Nutrition clinique pratique : chez l'adulte et l'enfant, Elsevier-Masson (ed). ElsevierMasson: Issy-les-Moulineaux; 189-205.

Shen J, Kondal D, Rubinstein A, Irazola V, Gutierrez L, Miranda JJ, Bernabé-Ortiz A, Lazo-Porras M, Levitt N, Steyn K, Bobrow K, Ali MK, Prabhakaran D, Tanon N.2016. A multiethnic study of pre-diabetes and diabetes in low and medium income countries. Global Heart, 11(1): 61-70. DOI: https://doi.org/10.1016/j.gheart.2015.12. 015

Suvi K, Joao de Roche F, Yadi H, Belama M. 2017. FID des Atlas du diabète ( ${ }^{8}$ ème Edn). IDF Communications : Brussels

World Health Organization. 2018. Noncommunicable disease country profiles 2014-2018

https://www.who.int/nmh/countries/tgo_ en.pdf (consulté le 11 mars 2020). 